\title{
Mapping Of Nanoscale Structural Fluctuations In Ferroelectric BaTiO 3 Using STEM-CBED
}

\section{Kenji Tsuda ${ }^{1}$}

1. Institute of Multidisciplinary Research for Advanced Materials, Tohoku University, Sendai, Japan.

The convergent-beam electron diffraction (CBED) method has become established as the most powerful technique for determining crystal symmetries of specimen areas of a few nanometers in diameter [e.g. 1]. The CBED method was extended to quantitative crystal structure analysis for determining structural parameters such as atom positions, atomic displacement parameters (ADPs) and electrostatic potential distributions [2-4]. To examine nanometer-scale variations of local structures, we proposed a combined use of scanning the transmission electron microscopy (STEM) and the CBED (STEM-CBED method). The STEM-CBED method enables us to visualize nanometer-scale spatial distributions of local structures with picometer-scale sensitivity to atom-displacements because of a full use of the twodimensional angular distributions of diffraction intensities [5].

The STEM-CBED experiments are conducted using a JEM-2010FEF transmission electron microscope equipped with an Omega-type energy filter and a STEM unit with a Gatan STEM diffraction imaging system. The energy filtering is very effective for reducing inelastically-scattered background intensities in CBED patterns. Energy-filtered CBED patterns are acquired pixel-by-pixel by scanning the convergent-beam electron probe with a sub-nanometer scan step [5]. A similar technique called scanning CBED was developed by Kim et al. [6].

We have applied the STEM-CBED method to perovskite-type ferroelectric oxides like $\mathrm{BaTiO}_{3}$. Despite extensive studies over many decades, the mechanism of their structural phase transformations has been a matter of discussion [e.g. 7, 8]. Examinations of their nanometer-scale local structures are crucial to resolve this problem. In the tetragonal phase of $\mathrm{BaTiO}_{3}$, two-dimensional distributions of nanoscale fluctuations of the rhombohedral polarization clusters were visualized from the STEM-CBED patterns [5]. It was revealed that the tetragonal phase of $\mathrm{BaTiO}_{3}$ is formed as the average structure of the variants of the rhombohedral nanostructures with different orientations of polarizations. Similar rhombohedral nanostructures were observed in the orthorhombic phase of $\mathrm{KNbO}_{3}$ [9]. These show the order-disorder character in the phase transformations. The scales of spatial correlations of the local polarizations can be related to the transition entropy [9].

It is also very important to investigate the local structures of the paraelectric cubic phase of $\mathrm{BaTiO}_{3}$ above the Curie temperature of $T_{\mathrm{c}}=403 \mathrm{~K}$ because precursors of the phase transformation are expected to exist in the cubic phase. Figures 1(a) and 1(b) show energy-filtered CBED patterns of the cubic phase of $\mathrm{BaTiO}_{3}$ taken at $415 \mathrm{~K}$ and $460 \mathrm{~K}$, respectively. It is seen that the pattern taken at $415 \mathrm{~K}$ exhibits clear breaking of the fourfold rotation symmetry expected from the cubic phase, while the pattern taken at $460 \mathrm{~K}$ shows almost the fourfold rotation symmetry. This indicates that there exist local polarization clusters in the cubic phase, and the polarization clusters are decreased with increasing temperature. A symmetry-breaking index of the CBED pattern was mapped from the STEM-CBED patterns. The details will be presented in the talk. [10] 


\section{References:}

[1] M. Tanaka and K. Tsuda, J. Electron Microsc. 60(Suppl. 1) (2011), p. S245.

[2] K. Tsuda and M. Tanaka, Acta Cryst. A55 (1999), p. 939.

[3] Y. Ogata, K. Tsuda and M. Tanaka, Acta. Cryst. A64, 587 (2008).

[4] K. Tsuda, D. Morikawa, Y. Watanabe, S. Ohtani, T. Arima, Phys. Rev. B 81 (2010), p. 180102.

[5] K. Tsuda, A. Yasuhara and M. Tanaka, Appl. Phys. Lett. 102 (2013) p. 051913.

[6] K. H. Kim, D. A. Payne and J. M. Zuo, J. Appl. Cryst. 46 (2013), p. 1331.

[7] R. Pirc and R. Blinc, Phys. Rev. B 70, (2004), p. 134107.

[8] A. Bussmann-Holder, H. Beige and G. Völkel, Phys. Rev. B 79 (2009), p. 184111.

[9] K. Tsuda and M. Tanaka, Appl. Phys. Express. 8, (2015), p. 081501.

[10] The present study was performed in collaboration with Emer. Prof. M. Tanaka and Mr. R. Sano (IMRAM, Tohoku University), and Mr. A. Yasuhara (JEOL Ltd.), and was partially supported by JSPS KAKENHI Grant Numbers 25287068 and 25630272. The author is grateful to Prof. M. Terauchi for his continuous support to this work.

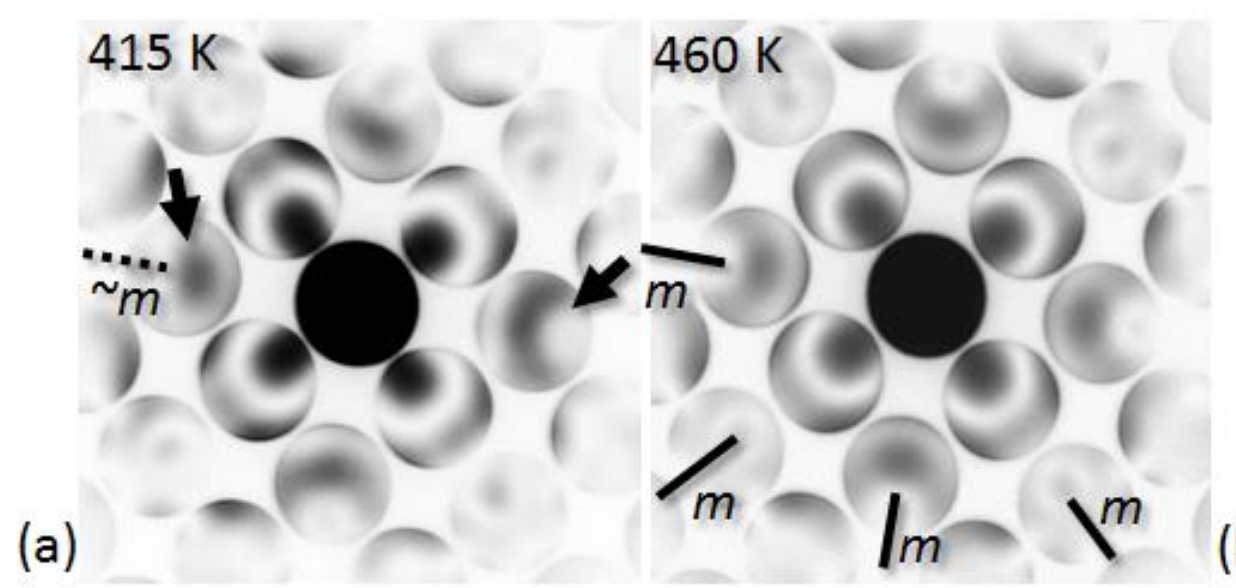

Figure 1. Energy-filtered [100] CBED patterns of the cubic phase of $\mathrm{BaTiO}_{3}$ taken at specimen temperatures of (a) $415 \mathrm{~K}$ and (b) $460 \mathrm{~K}$. The pattern (a) exhibit clear breaking of the fourfold rotation symmetry expected from the cubic phase, while the pattern (b) shows almost the fourfold rotation symmetry. 